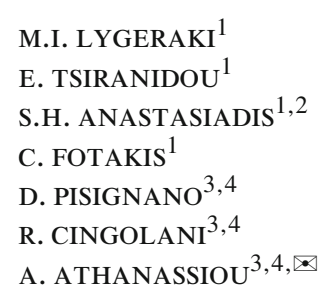

\section{Controlling the reversible wetting capability of smart photochromic-polymer surfaces by micro patterning}

\author{
${ }^{1}$ Institute of Electronic Structure and Laser (IESL), Foundation for Research and Technology - Hellas \\ (FORTH), Heraklion, Crete, Greece \\ 2 Aristotle University of Thessaloniki, Department of Chemical Engineering, 54124 Thessaloniki, Greece \\ ${ }^{3} \mathrm{NNL}$ - National Nanotechnology Laboratory, INFM, CNR, Via Arnesano, 73100 Lecce, Italy \\ ${ }^{4}$ IIT - Italian Institute of Technology, Via Morego 30, 16163 Genova, Italy
}

\begin{abstract}
Received: 30 January 2008/Accepted: 25 February 2008 Published online: 29 March 2008 • (C) Springer-Verlag 2008

ABSTRACT We demonstrate the wetting behavior control of polymer surfaces doped with photochromic molecules by modifying the surface patterning features introduced by soft molding lithography. Such surfaces enhance their hydrophilicity upon UV irradiation due to conversion of the non-polar spiropyran dopant molecules to their polar merocyanine isomers. The process is reversed upon visible light irradiation. By changing the topological parameters of the introduced pattern, one achieves surface tuning from hydrophobic to hydrophilic situations. The difference for the contact angles between UVand green-irradiated surfaces may become significantly higher than for the flat surfaces, for the specific patterning parameters analyzed.
\end{abstract}

PACS 42.62.-b; 68.08.Bc; 83.50.Uv; 42.70.Jk; 42.70.Gi

1

\section{Introduction}

The fabrication and understanding of smart surfaces with reversible and controlled wetting properties, responsive to external stimuli [1-6], can enhance the functionality of many systems relevant for controllable drug delivery [7], actuators [8], microfluidic devices [9], and selfcleaning surfaces [10]. In this work, we examine the effect of micropatterning on the wettability of smart surfaces of polymers incorporating photochromic molecules, and its reversible modification upon alternating UV and visible irradiation. We demonstrate the possibility of tuning a patterned surface from very hydrophobic to hydrophilic by changing its structural characteristics, and we present the control of the water contact angle differences between UV and visible light irradiated samples by manipulating the patterning parameters.

In previous works we demonstrated that polymers incorporating photochromic spiropyran (SP) molecules exhibit reversible wettability changes in response to the external stimulus of light [11-13]. This behavior is due to the light-induced transformations of the dopant molecule. The SP molecule is colorless and non-polar, but, after absorption of UV photons,

Fax: +39-0832-298238, E-mail: athanassia.athanassiou@unile.it is converted to its colored and polar isomeric merocyanine (MC) form. The conversion occurs due to the photochemical cleavage of the $\mathrm{C}-\mathrm{O}$ bond in the SP ring and the consequent ring opening [14], as demonstrated in Fig. 1. This isomerization process is reversible, with the MC being converted back to the SP upon irradiation with visible light [15]. The photochromic molecules undergo the reversible isomerization process even when incorporated within macromolecular matrices [16-18], interchanging between the non-polar SP and the polar MC form. This change induces an overall change in the polarity of their host polymers [19,20], thus modifying also their surface polarity. As a result, the surfaces exhibit enhanced hydrophilicity after UV irradiation and enhanced hydrophobicity after visible light irradiation $[11,12]$. Concurrently, the host matrices also respond to the light-induced photochromic transformations by reversibly changing their macroscopic dimensions [21]. In particular, after the first UV irradiation pulses, the formed MC stereoisomers tend to aggregate reducing the $\mathrm{MC}$ partial molar volume. Consequently, short scale motion of the polymer chains occurs in order to diminish the density fluctuations in the samples, and this leads to the macroscopic reduction of the dimensions of the matrix. The dimensions are recovered upon visible irradiation due the return of the MC molecules to the initial SP form, which does not form aggregates. Thus, the polymer chains are forced to return to their initial positions [21]. This property plays a crucial role in the wetting characteristics of structured polymer-spiropyran surfaces, by changing the dimensions of the structured features and, subsequently, the overall surface topography [11].

\section{$2 \quad$ Experimental details}

The samples consist of poly(ethyl methacrylate)co-poly(methyl acrylate), P(EMA)-co-P(MA), with average MW $\sim 100000$ (Aldrich) and glass transition temperature $T_{\mathrm{g}}=48^{\circ} \mathrm{C}$, and photochromic molecules $1^{\prime}, 3^{\prime}$-dihydro- $1^{\prime}, 3^{\prime}$, $3^{\prime}$-trimethyl-6-nitrospiro(2H-1-benzopyran-2,2'-2H-indole), commonly referred as 6- $\mathrm{NO}_{2}$ BIPS, used as an additive at $10 \mathrm{wt}$ \%. The flat films were prepared by spin coating toluene solutions on glass substrates. The patterning of their surface was achieved using soft molding [22], a powerful technique to pattern organic materials [23]. Three elastomeric replicas, 


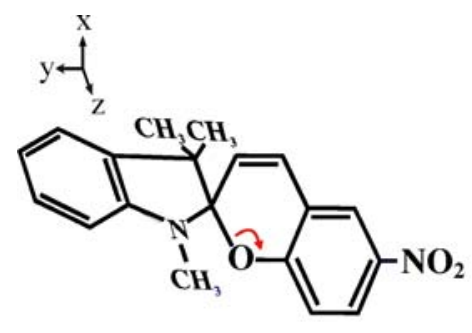

Spiropyran
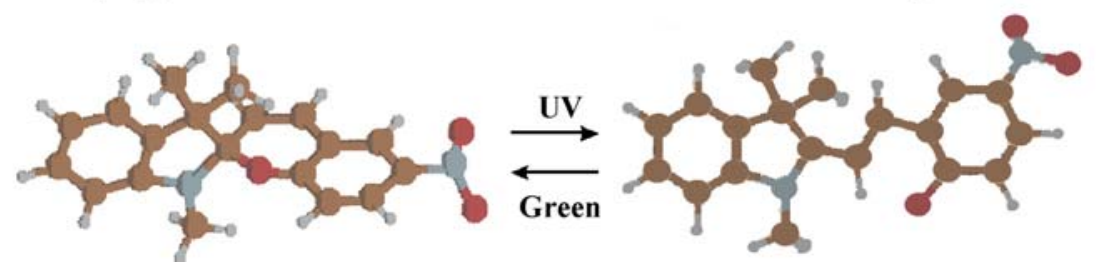

FIGURE 1 Spiropyran/merocyanine isomers (graphical representation) fabricated starting by three symmetric master gratings with periods of $1.3,28.0$, and $180.0 \mu \mathrm{m}$, were positioned onto the spin coated SP-polymer films under their own weight at $50{ }^{\circ} \mathrm{C}$. After a few minutes the patterns were faithfully transferred on the films and the replicas were peeled off. During the patterning procedure the temperature of the samples should always be kept close to $50^{\circ} \mathrm{C}$, since it was observed that, as the temperature increases well above the $T_{\mathrm{g}}$ of the system, the doped photochromic molecules tend to migrate out of the polymer matrix and move onto the replicas. The exact parameters of the prepared patterned samples were established using atomic force and scanning electron microscopy.

Drops of $3 \mu \mathrm{l}$ of millipore water (18.2 ME) were used for the contact angle measurements. After the measurement of the initial contact angle, the drop was removed and the sample was irradiated with $50 \mathrm{UV}$ pulses of a $\mathrm{XeCl}$ laser, operating at $308 \mathrm{~nm}, \tau_{\text {pulse }} \sim 30 \mathrm{~ns}$, and fluence $20 \mathrm{~mJ} \mathrm{~cm}^{-2}$. The new contact angle of a water drop was measured after the UV irradiation. The sample was then again dried and irradiated with 200 laser pulses of a Nd:YAG laser, operating at the second harmonic, $532 \mathrm{~nm}, \tau_{\text {pulse }} \sim 5 \mathrm{~ns}$, and fluence $25 \mathrm{~mJ} \mathrm{~cm}^{-2}$. A new water contact angle was measured after the green irradiation, etc. The laser beams were overlapping onto the surface of the samples at an area of $3.5 \times 3.5 \mathrm{~mm}^{2}$. The number and the energy density of the UV laser pulses were chosen after careful spectroscopic studies, so that complete conversion of the SP molecules to their MC isomeric form is ensured. Additional laser pulses do not influence the wetting of the surfaces any further.

\section{Results and discussion}

On each sample typically 5-8 irradiation cycles can be performed with good reversibility in its wetting behavior, since the photochromic molecules undergo degradative photooxidation, which limits the lifetime of the system [24]. Usually degradative phenomena start to be evident after the third cycle. Furthermore, statistically we observed an increased possibility for different and random wetting behavior of some samples upon the first irradiation cycle compared to all the subsequent cycles. This can be explained by the fact that possible internal stresses, accumulated in the ma- trix upon the preparation of the samples, are released during the first irradiation cycle through rearrangement of the polymer chains, which leads to the equilibrium conformation of the system [25]. The release of these stresses affects the morphology and thus the wettability of the pattern surfaces. To exclude the parameters of the stresses and of the degradation from the evaluation of the surface topological parameters on the wetting properties of the surface we examine only the second and the third irradiation/wetting cycles on the patterned samples. The periods of 1.3, 28.0, and $180.0 \mu \mathrm{m}$ of the surface gratings used in this study were confirmed by atomic force and scanning electron microscopy measurements. Typical patterned surfaces are presented in Fig. 2.

Contact angles differences of a few degrees can be monitored between surfaces patterned with the same replica, due to different imprinting quality. In Fig. 3 a the mean values of the contact angles are plotted of ten different samples for each patterning period, and of at least ten flat samples. Moreover, the average values of the contact angles of water drops on a flat polymer surface without SP molecules are also presented for comparison (continuous line). In this case laser irradiation has no impact on the contact angles, since the light-induced interconversions of the doped photochromic molecules are exclusively responsible for the changes in the wetting properties. Figure 3 a demonstrates that any patterning enhances the hydrophobicity of the surfaces compared to the flat ones. This behavior is described by the Cassie and Baxter model [26], originally developed to describe the wettability of rough surfaces, where only partial wetting may occur due to the trapping of air underneath the drop at the recessed regions of the surfaces. In our case the pattern is nothing but an ordered roughness, and the contact angle, $\theta_{\mathrm{r}}$, is an average between the value on air $\left(180^{\circ}\right)$ and the value on the flat surface $\theta$. Since the drop is situated partially on air, the rough (patterned) surface always exhibits an increased contact angle compared to the corresponding flat surface. The model reads

$\cos \theta_{\mathrm{r}}=f(1+\cos \theta)-1$,

where $f \leq 1$ is the solid fraction of the surface in contact with the liquid. 

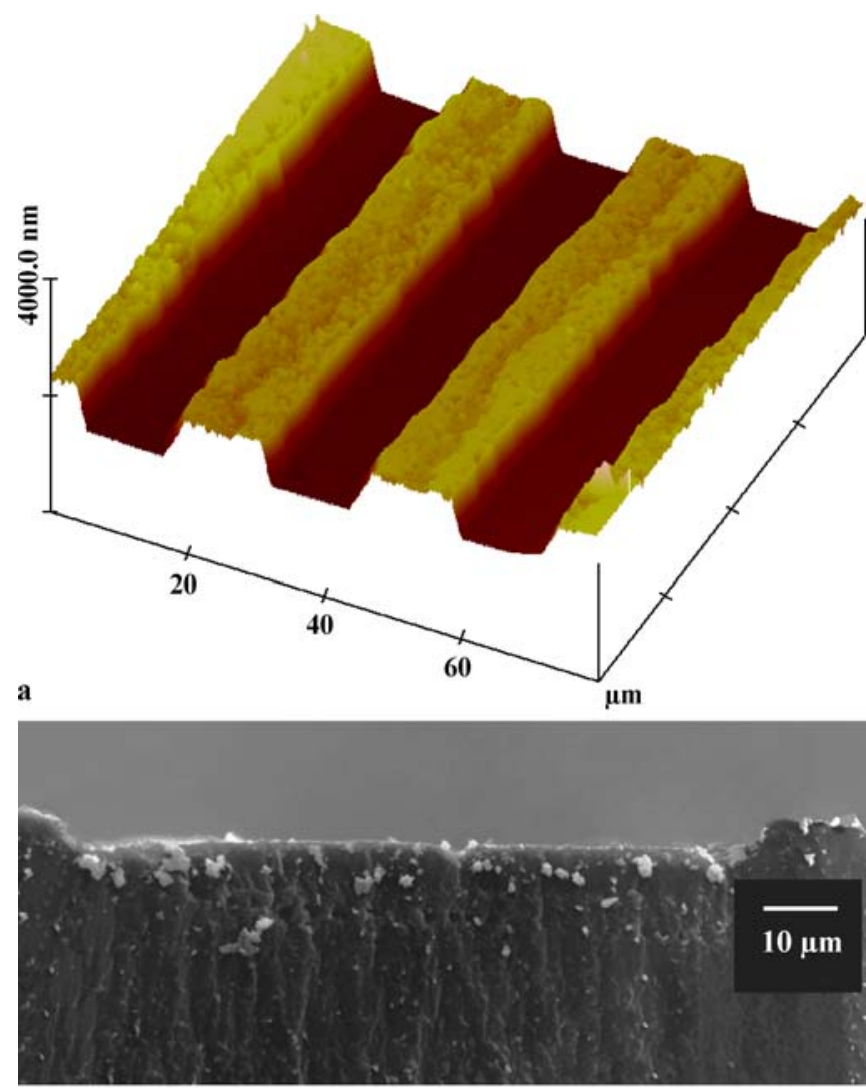

b

FIGURE 2 Nanoimprinted gratings on the surfaces of a 10\% SP-90\% $\mathrm{P}(\mathrm{EMA})-\mathrm{co}-\mathrm{P}(\mathrm{MA})$ samples shown by images of atomic force microscopy (period of $28 \mu \mathrm{m})(\mathbf{a})$. The $x$ and $y$ axis are presented at a different scale. (b) Scanning electron microscopy image of a cross section of a sample with a $180 \mu \mathrm{m}$ period

There are articles that question the general validity of the Cassie-Baxter model, since they demonstrate that the contact angles are determined by interactions of the liquid and the solid at the three-phase contact line, and not at the interfacial contact area within the contact perimeter $[27,28]$. Nevertheless, the Cassie-Baxter model is valid when the structure of the contact area reflects that of the contact line, which is the case in our samples, where the structure uniformly continues in and out of the drop area. Figure $3 b$ demonstrates that the differences in the surface wetting between alternating irradiations increase for decreasing period patterns. This cannot be attributed to enhanced polarity differences between UV and green-irradiated surfaces in the case of the small period patterning since the surface chemistry is the same for all the samples. Moreover, surface chemistry alterations should affect more the samples with high period patterns, since in these cases the drops are in contact with a greater percentage of the solid surface. This is demonstrated by the increasing value of the factor $f$ of the Cassie-Baxter model as the period increases; this is presented in Table 1.

Therefore, the big differences in the contact angles in the case of small period patterns are attributed to the shrinkage of the protruding features upon UV irradiation [11,21], caused by the mechanism mentioned in the introduction. Due to this shrinkage the water drops penetrate deeper into the channels of the patterned gratings increasing the fraction for the sur-
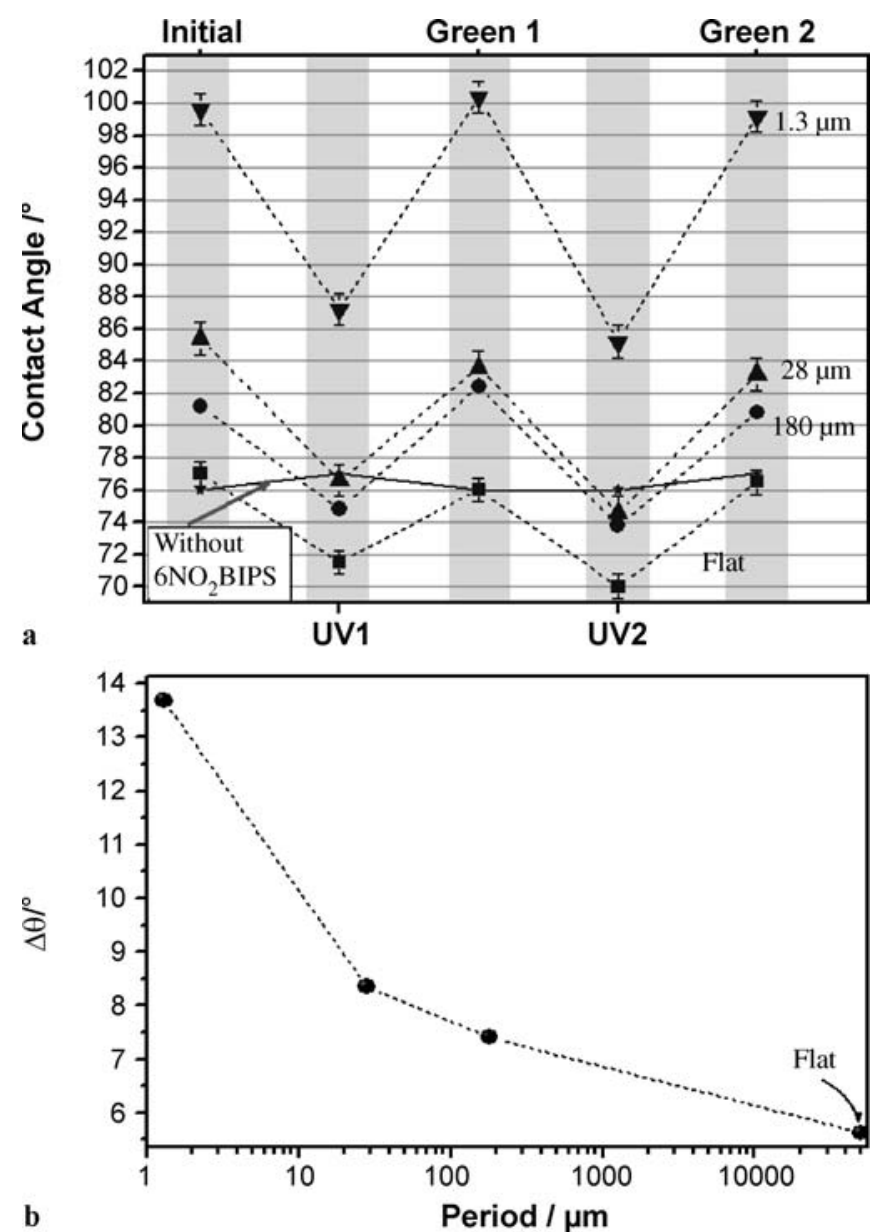

FIGURE 3 (a) Average contact angle values of water drops situated on the initial, the UV irradiated, and the green irradiated surfaces of $10 \%$ SP-90\% $\mathrm{P}(\mathrm{EMA})$-co-P(MA) samples, flat (ם) and patterned with periods of $1.3 \mu \mathrm{m}$ $(\mathbf{\nabla}), 28.0 \mu \mathrm{m}(\mathbf{\Delta})$, and $180.0 \mu \mathrm{m}(\mathbf{\bullet})$. (b) Average contact angle differences between alternating irradiations for each patterning period and for the flat samples

\begin{tabular}{lccc}
\hline Period & $1.3 \mu \mathrm{m}$ & $28 \mu \mathrm{m}$ & $180 \mu \mathrm{m}$ \\
\hline Initial & 0.68 & 0.88 & 0.94 \\
UV1 & 0.80 & 0.94 & 0.96 \\
GREEN1 & 0.66 & 0.89 & 0.91 \\
UV2 & 0.81 & 0.94 & 0.95 \\
GREEN2 & 0.68 & 0.91 & 0.94 \\
\hline
\end{tabular}

TABLE 1 Factor $f$ calculated by the Cassie-Baxter model for the three differently patterned samples under different irradiation conditions

face that is wetted. This contraction influences much more the contact angle of the samples with patterns with small period because the drop lies on a greater number of patterned features. It is calculated that the radius of the falling drop is 20 times larger than the size of each feature projected on the plane of the interface in the case of the patterns with a $180 \mu \mathrm{m}$ period, about 140 times greater in the case of the patterns with a $28 \mu \mathrm{m}$ period, and finally more that 2950 times greater in the case of the patterns with a $1.3 \mu \mathrm{m}$ period. Indeed, from the value $f$ in Table 1 it is clear that for patterning with a $180 \mu \mathrm{m}$ period gratings, the percentage of the surface that is wetted changes slightly $(1 \%-5 \%)$ between the alternating irradiations. For the patterns with a period of $28.0 \mu \mathrm{m}$, the fraction of 


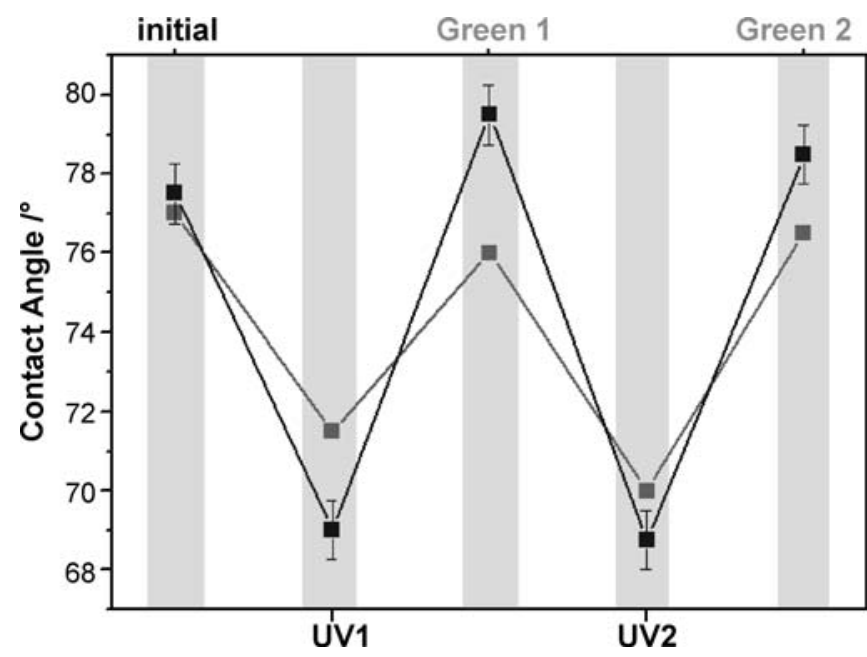

FIGURE 4 Average contact angle values of water drops situated on the initial, the UV irradiated, and the green-irradiated surfaces of ( $\square$ ) samples patterned with a $180 \mu \mathrm{m}$ period that exhibit partial and complete wetting reversibly between the alternating irradiations; ( $\square$ ) flat samples

the water in contact with the solid surface can increase up to $6 \%$ from the UV to the green-irradiated surfaces. Finally, for the patterns with a period of $1.3 \mu \mathrm{m}$, the fraction of the features that are wetted after UV irradiation can be up to $15 \%$ higher that the one after green irradiation.

Interestingly, samples with a wide period and particularly smooth sidewalls of the pattern (such as those obtained with new masters; see Fig. 2b) result in a very good adhesion of the drop even inside the grooves of the pattern, with little (or no) air trapped under the drop. Such a condition was observed in a few patterned samples of period $180 \mu \mathrm{m}$, which exhibited a very interesting surface wetting behavior upon irradiation cycles: before any irradiation or after green irradiation they were partially wetted with contact angles higher than the ones for flat samples. Nevertheless, after UV irradiation the water contact angles were lower than the ones obtained on the flat samples. Their mean contact angle values are demonstrated in Fig. 4.

In these cases, after UV irradiation, we can assume that complete wetting of the patterned surfaces occurs, and therefore, the liquid-solid interfacial area is increased. Such a situation is more appropriately described by the Wenzel model [29], which predicts that when the contact angle of the flat surface is $\theta<90^{\circ}$ (like in our samples), and the water contact angle of the rough (patterned) surface is lower than the one of the flat surface. That is,

$\cos \theta_{\mathrm{r}}=r \cos \theta$,

where $\theta$ is the contact angle on the flat surface of the same nature and $r$ is the surface roughness defined as the ratio of the actual wetted surface over the surface as measured on the plane of the interface (in general $r>1$, and $r=1$ for flat surfaces).

Calculating the values of $r$ from the Wenzel equation for our samples after UV irradiation and substituting them in the equation for the roughness $r=(T+2 d) / T$, where $d$ is the depth of the grating and $T$ its period, we estimate the depth of the grooves $\sim 5-12 \mu \mathrm{m}$ for the gratings with $180.0 \mu \mathrm{m}$ period. Indeed, these values are in the range of the values measured with scanning electron microscopy.

Such a switching behavior between partial and complete wetting, which we achieve exclusively by light irradiation of the surfaces, is of great interest for micro/nanofluidic applications, and it has been accomplished by other groups by applying mechanical pressure on the drop [30]. The samples that exhibited this switching behavior were the ones with a minimum surface micro-roughness within the patterned features (one of them is shown in Fig. 2b). Further investigation for stabilizing the patterning parameters of such samples is in progress. This kind of samples can in principle exhibit very large contact angle changes between alternating irradiations by increasing the roughness parameter $r$ in the Wenzel model, i.e. increasing the depth of the grooves of the patterned features, resulting in very low contact angles after UV irradiation.

\section{$4 \quad$ Conclusions}

Summarizing, we presented the possibility to create both hydrophobic and hydrophilic surfaces starting from the same photochromic-polymeric sample by changing the topological parameters of its surface features. Due to the photochromic transformations taking place upon alternating UV and green irradiation, these surfaces can reversibly change their wettability. By careful control of the surface topology these changes can be fully controlled and tuned, even in such a way that the surfaces can be partially or fully wetted in a reversible manner.

ACKNOWLEDGEMENTS The authors thank Mrs. A. Manousaki (IESL-FORTH, Heraklion, Crete, Greece) for her valuable help in the Atomic Force Microscopy measurements.

\section{REFERENCES}

1 S.T. Wang, Y.L. Song, L. Jiang, J. Photochem. Photobiol. C 8, 18 (2007)

2 R. Rosario, D. Gust, A.A. Garcia, M. Hayes, J.L. Taraci, T. Clement, J.W. Dailey, S.T. Picraux, J. Phys. Chem. B 108, 12640 (2004)

3 J. Lahann, S. Mitragotri, T.-N. Tran, H. Kaido, J. Sundaram, I.S. Choi, S. Hoffer, G.A. Somorjai, R. Langer, Science 299, 371 (2003)

4 B. Coupe, M.E. Evangelista, R.M. Yeung, W. Chen, Langmuir 17, 1956 (2001)

5 S.H. Anastasiadis, H. Retsos, S. Pispas, N. Hadjichristidis, S. Neophytides, Macromolecules 36, 1994 (2003)

6 J. Isaksson, C. Tengstedt, M. Fahlman, N. Robinson, M. Berggren, Adv. Mater. 16, 316 (2004)

7 D.A. La Van, T. McGuire, R. Langer, Nat. Biotechnol. 21, 1184 (2003)

8 A. Sidorenko, T. Krupenkin, A. Taylor, P. Fratzl, J. Aizenberg, Science 315, 487 (2007)

9 H. Nagai, T. Irie, J. Takahashi, S.-I. Wakida, Biosens. Bioelectron. 22, 1968 (2007)

10 X. Zhang, A. Fujishima, M. Jin, A.V. Emeline, T. Murakami, J. Phys. Chem. B 110, 25142 (2006)

11 A. Athanassiou, M.I. Lygeraki, D. Pisignano, K. Lakiotaki, M. Varda, E. Mele, C. Fotakis, R. Cingolani, S.H. Anastasiadis, Langmuir 22, 2329 (2006)

12 A. Athanassiou, M. Varda, E. Mele, M.I. Lygeraki, D. Pisignano, M. Farsari, C. Fotakis, R. Cingolani, S.H. Anastasiadis, Appl. Phys. A 83, 351 (2006)

13 E. Mele, D. Pisignano, M. Varda, M. Farsari, G. Filippidis, C. Fotakis, A. Athanassiou, R. Cingolani, Appl. Phys. Lett. 88, 203124 (2006)

14 R.C. Berthelson, Photochromism, ed. by G.H. Brown (Wiley-Interscience, New York, 1971), p. 45-294

15 A.K. Chibisov, H. Görner, J. Photochem. Photobiol. A 105, 261 (1997) 
16 G. Smets, Adv. Polym. Sci. 50, 17 (1983)

17 A. Tork, F. Boudreault, M. Roberge, A.M. Ritcey, R.A. Lessard, T.V. Galstian, Appl. Opt. 40, 1180 (2001)

18 H. Bouas-Laurent, H. Durr, Pure Appl. Chem. 73, 639 (2001)

19 K. Kimura, H. Sakamoto, T. Nakamura, J. Nanosci. Nanotechnol. 6, 1741 (2006)

20 A. Fritz, A. Schönhals, B. Sapich, J. Stumpe, Macromol. Chem. Phys. 200, 2213 (1999)

21 A. Athanassiou, M. Kalyva, K. Lakiotaki, S. Georgiou, C. Fotakis, Adv. Mater. 17, 988 (2005)

22 K.Y. Suh, Y.S. Kim, H.H. Lee, Adv. Mater. 13, 1386 (2001)
23 D. Pisignano, L. Persano, G. Gigli, R. Cingolani, F. Babudri, G.M. Farinosa, F. Naso, Appl. Phys. Lett. 84, 1365 (2004)

24 A. Athanassiou, D. Sahinidou, V. Arima, S. Georgiou, R. Cingolani, C. Fotakis, J. Photochem. Photobiol. A 183, 182 (2006)

25 G.-D. Liang, J.-T. Xu, Z.-Q. Fan, S.-M. Mai, A.J. Ryan, Langmuir 23, 3673 (2007)

26 A.B.D. Cassie, S. Baxter, Trans. Faraday Soc. 40, 546 (1944)

27 C.W. Extrand, Langmuir 19, 3793 (2003)

28 L. Gao, T.J. McCarthy, Langmuir 23, 3762 (2007)

29 R.N. Wenzel, Ind. Eng. Chem. 28, 988 (1936)

30 A. Lafuma, D. Quéré, Nature Mater. 2, 457 (2003) 